\title{
Treatment outcomes of T3 laryngeal carcinomas - surgical versus non-surgical management: a 10-year multicentre audit in the northeast of England.
}

\author{
Daniel J Lin', Michael Goodfellow², Jen Ong ${ }^{3}$, Mei Ying Chin ${ }^{4}$, Lepa Lazarova ${ }^{4}$, Helen C Cocks ${ }^{1}$
}

\section{Introduction}

Larynx preservation remains an important treatment goal for T3 laryngeal carcinomas. Treatment options involving chemoradiotherapy (CRT) are often preferred over laryngectomy. However, treatment-related toxicity from nonsurgical options may result in a dysfunctional larynx requiring salvage laryngectomy. We aim to compare three institutional treatment outcomes for patients with T3 laryngeal carcinomas to ascertain the optimal management strategies.

\section{Methods}

A retrospective audit on the management of pre-treatment diagnosed T3 laryngeal carcinomas. Patient cases were selected from three institutions between the years 2007-2016. Case note review was performed using electronic medical records and descriptive statistics obtained. Locoregional control and overall survival (OS) rates were compared between the treatment regimens using Pearson chi-square test and KaplanMeier survival curves, respectively.

Figure I. Descriptive statistics and patient demographics

- 179 patients, 133 male and 46 female

- Male - Mean age $=65.65$
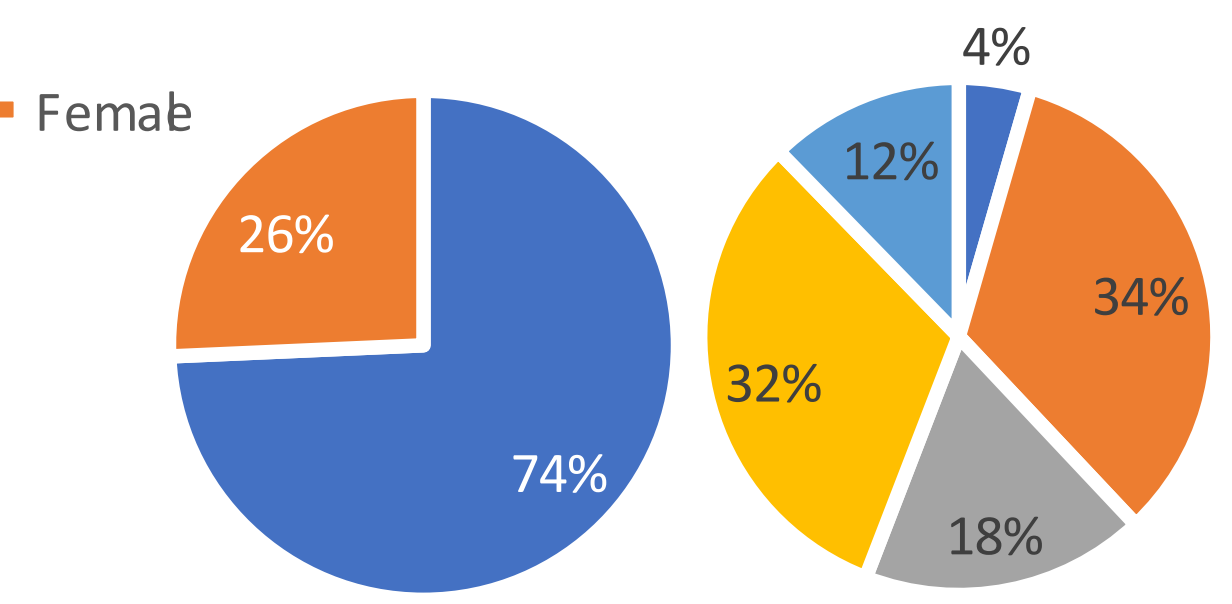

- Partial

La yngectomy La yngetomy

\section{- RT}

CRT

- Pallat ive

Table I. Locoregional recurrences by treatment regimen

\begin{tabular}{cccccc|} 
Recurrences & $\begin{array}{c}\text { Number of } \\
\text { patients }\end{array}$ & $\begin{array}{c}\text { Number of } \\
\text { recurrences }\end{array}$ & $\%$ & Local & Distant \\
\hline Laryngectomy & 68 & 15 & 22.1 & 9 & 6 \\
\hline CRT & 57 & 10 & 17.5 & 7 & 3 \\
\hline RT & 32 & 16 & 50.0 & 11 & 5 \\
\hline
\end{tabular}

Pearson-Chi squared comparison of recurrence:

- Laryngectomy vs CRT: $\quad P=0.53$

-Laryngectomy vs RT: $\quad P=0.005$

-CRT vs RT: $\quad P=0.001$

\section{Correspondence:}

Daniel J Lin, BSc (Hons), MBChB

NIHR Academic Clinical Fellow in ENT

ENT Department, Sunderland Royal Hospital

E-mail: daniel.lin@newcastle.ac.uk

\section{Results}

There were 179 T3 laryngeal carcinomas (68 laryngectomies, 57 CRT, 32 radiotherapy). No significant 5-year survival difference between laryngectomy (20.6\%) and CRT (29.8\%), $\mathrm{p}=0.184$. Five-year OS for RT (9.4\%); significantly inferior compared to laryngectomy and CRT $(p=0.003, p<0.00 I)$, respectively. Recurrence rates: $22.1 \%$ laryngectomy, $17.5 \%$ CRT and $50 \%$ radiotherapy, significantly different comparing laryngectomy $(p=0.005)$ and CRT $(p=0.00 \mathrm{I})$ to radiotherapy.

\section{Conclusions}

Laryngectomy and CRT had significantly higher 5-year OS and lower recurrence rates compared with radiotherapy alone. It is important to consider laryngectomy in patients who are unsuitable for chemotherapy since it may convey a significant survival advantage over radiotherapy alone.

Figure 2. Kaplan-Meier survival curves: 5-yr overall survival
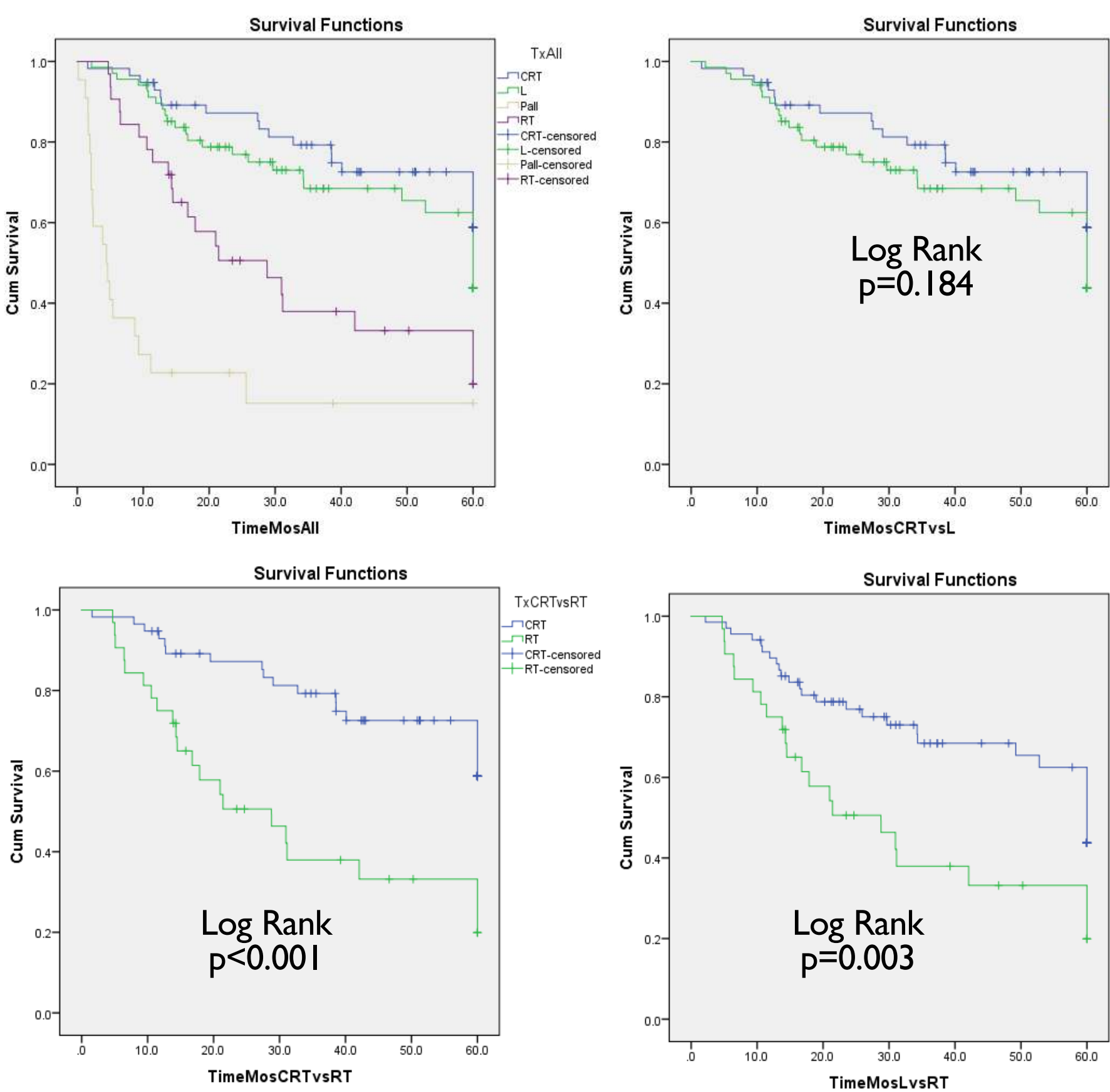

Table 2. Salvage laryngectomy rates

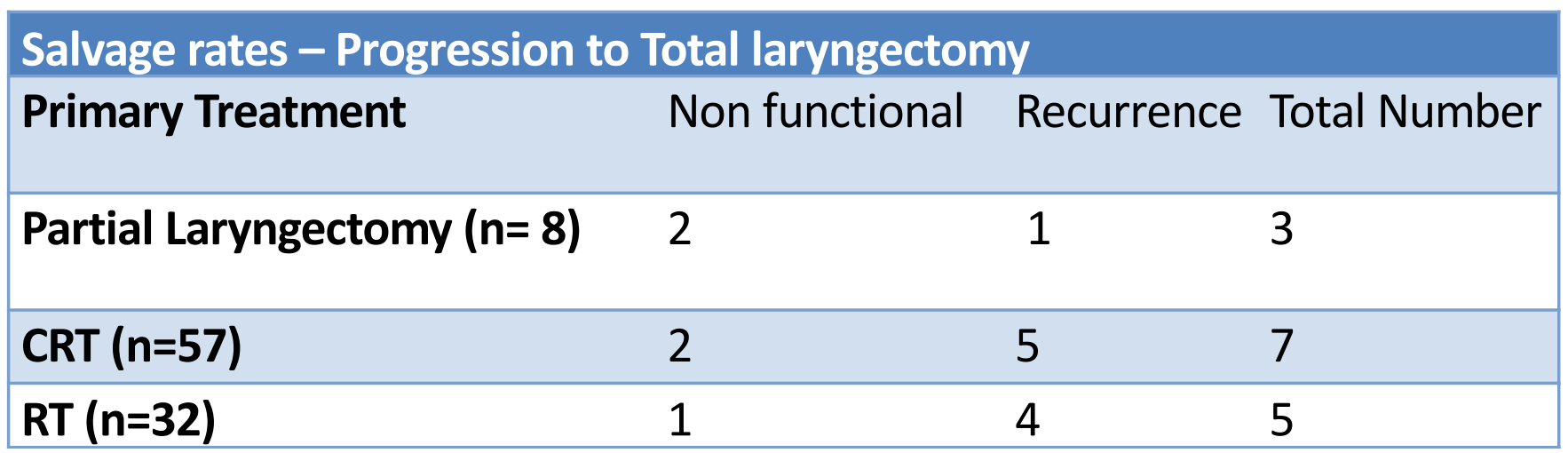

I. ENT Department, City Hospitals Sunderland NHS Foundation Trust, Sunderland, UK 2. Newcastle Medical School, Newcastle University, Newcastle upon Tyne, UK

3. ENT Department, Newcastle Upon Tyne Hospitals NHS Foundation Trust 4. ENT Department, North Cumbria University Hospital, Carlisle, UK 